\title{
Characterization and virulence clustering analysis of extraintestinal pathogenic Escherichia coli isolated from swine in China
}

Yinchu Zhu ${ }^{1,2 \dagger}$, Wenyang Dong ${ }^{1,2 \dagger}$, Jiale Ma ${ }^{1,2}$, Lvfeng Yuan ${ }^{1}$, Hassan M.A. Hejair ${ }^{1,2}$, Zihao Pan ${ }^{1,2}$, Guangjin Liu ${ }^{1,2}$ and Huochun Yao ${ }^{1,2^{*}}$

\begin{abstract}
Background: Swine extraintestinal pathogenic Escherichia coli (ExPEC) is an important pathogen that leads to economic and welfare costs in the swine industry worldwide, and is occurring with increasing frequency in China. By far, various virulence factors have been recognized in ExPEC. Here, we investigated the virulence genotypes and clonal structure of collected strains to improve the knowledge of phylogenetic traits of porcine ExPECs in China.

Results: We isolated 64 Chinese porcine ExPEC strains from 2013 to 14 in China. By multiplex PCR, the distribution of isolates belonging to phylogenetic groups B1, B2, A and D was $9.4 \%, 10.9 \%, 57.8 \%$ and $21.9 \%$, respectively. Nineteen virulence-related genes were detected by PCR assay; ompA, fimH, vat, traT and iutA were highly prevalent. Virulence-related genes were remarkably more prevalent in group B2 than in groups $A, B 1$ and D; notably, usp, cnf1, hlyD, papA and ibeA were only found in group B2 strains. Genotyping analysis was performed and four clusters of strains (named I to IV) were identified. Cluster IV contained all isolates from group B2 and Cluster IV isolates had the strongest pathogenicity in a mouse infection model. As phylogenetic group B2 and D EXPEC isolates are generally considered virulent, multilocus sequence typing (MLST) analysis was performed for these isolates to further investigate genetic relationships. Two novel sequence types, ST5170 and ST5171, were discovered. Among the nine clonal complexes identified among our group B2 and D isolates, CC12 and CC95 have been indicated to have high zoonotic pathogenicity. The distinction between group B2 and non-B2 isolates in virulence and genotype accorded with MLST analysis.
\end{abstract}

Conclusion: This study reveals significant genetic diversity among ExPEC isolates and helps us to better understand their pathogenesis. Importantly, our data suggest group B2 (Cluster IV) strains have the highest risk of causing animal disease and illustrate the correlation between genotype and virulence.

Keywords: Porcine extraintestinal pathogenic Escherichia coli, Genotype, Virulence, Multilocus sequence typing

\section{Background}

Extraintestinal pathogenic Escherichia coli (ExPEC) is a major pathogenic agent causing disease worldwide. It can lead to many kinds of extraintestinal diseases, including newborn meningitis, sepsis, and urinary tract disease [1]. It has been reported to cause death in animals and humans in Europe and North America [2, 3].

\footnotetext{
*Correspondence: yaohch@njau.edu.cn

${ }^{\dagger}$ Equal contributors

${ }^{1}$ College of Veterinary Medicine, Nanjing Agricultural University, Nanjing 210095, China

${ }^{2}$ Key Lab of Animal Bacteriology, Ministry of Agriculture, Nanjing Agricultural University, Nanjing 210095, China
}

ExPEC can be distinguished from intestinal pathogenic E. coli and commensal E. coli by isolation site and genetic patterns [4]. Based on the isolation site, ExPEC can be classified as uropathogenic E. coli (UPEC), avian pathogenic E. coli (APEC), newborn meningitis E. coli (NMEC) or sepsis E. coli (SEPEC). The infectivity of ExPEC involves various extraintestinal virulenceassociated factors in microbe-host interactions, rather than a simple mechanism [5]. Genome plasticity of ExPEC is the basis for rapid adaptation to the changing environment, which in turn increases the diversity of $E$. coli populations. 
ExPEC is a major pathogen in the swine industry, responsible for considerable economic losses [6]. Alongside rapid development of industrial swine husbandry in China, an increasing trend of porcine ExPEC outbreaks has become an urgent problem [7]. Furthermore, the similarities in ExPEC isolates from human and animal infection cases suggest that there is cross-infection potential between different hosts, including humans, companion animals, pigs and birds [8]. I.e., ExPEC has high zoonotic potential, which makes porcine ExPEC a significant danger to public health.

E. coli consists of phylogenetic groups, namely A, B1, B2 and D. Most virulent ExPEC strains belong to group B2, followed by Group D. Generally, isolates assigned to groups A or B1 are less likely to cause disease in healthy animals [9]. The presence of virulence-related (VR) genes has a close connection with the virulence phenotype. Many genes have been identified as VR in ExPEC, and are involved in adhesion (P, S fimbriae, F1C fimbriae, Dr-binding adhesin and type I fimbriae), invasion (ibeA, invasive of brain endothelium), iron acquisition (siderophores), toxicity (hemolysin, vacuolating toxin, cytotoxic necrotizing factor) and surface protection (capsule) [10]. To identify combinations of VR genes associated with disease, the virulence genotyping method has previously been used in studies of UPEC [11]. However, such data is still limited for porcine ExPEC. In this study, we performed virulence genotyping, animal infection experiments and multilocus sequence typing (MLST) analysis, to provide insight into the associations between genotypes, phylogenetic groups and virulence phenotypes of ExPEC.

\section{Results}

Hemolytic activity tests

Of 64 tested ExPEC isolates, five produced $\beta$-hemolysin in $7 \%$ sheep blood agar, namely strains DCE-1, DCE-2, DCE-5, DCE-6, DCE-9.

\section{Prevalence of VFs}

The prevalence of VR genes was diverse (Additional file 1). iutA, vat, fimH, traT and ompA appeared in $>60 \%$ of strains (ompA was observed in all isolates). Among adhesion factors, fim $H$ was the most prevalent (81.2\%). Among siderophore factors, iutA (60.9\%) was more frequently detected than $f y u A$, ireA and iroN. The prevalence of cnfl, focG, ibeA, kpsMIII, afa, hlyD, $s f a A$, papA was $<10 \%$, and foc $G$ was not detected in any isolate. Table 1 shows these results in detail.

Phylogenetic group classification revealed that 9.4\% (6/64) of the porcine ExPEC isolates belonged to group B1, $10.9 \%(7 / 64)$ to group B2, $57.8 \%(37 / 64)$ to group A and 21.9\% (14/64) to group D. The phylogenetic group classification was associated with genetic patterns. The average number of VR genes in phylogenetic group B2 (12.1) was significantly higher than that in groups A (4.4), B1 (3.8), and D (4.3) (Fisher test, $P<0.05)$. hlyD, ibeA, usp, cnf1, papA were only observed in group B2 and $s f a A$, afa were only detected in group A. ireA, papA, sfaA, afa, kpsMIII, ibeA were rarely detected in any of the phylogenetic groups. Additional file 2: Table S1 shows statistical analysis of associations between virulenceassociated factors.

\section{Cluster structure analysis}

Based on virulence gene profiles, four virulence clusters (I, II, III, IV) were identified using BioNumerics software according to a previous method (Fig. 1). Seven strains belonged to Cluster I, with an average of 2.43 virulence-associated genes per strain. Four stains belonged to Cluster II, with average of 2.7 virulence-associated genes. Cluster III contained 46 isolates, with an average of 4.92 virulence-associated genes. Cluster IV contained seven isolates with an average 12 virulence-associated genes. In general, the virulence of phylogenetic group B2 E. coli isolates is considered to be highest, followed by group D, which is in turn higher than that of phylogenetic groups $\mathrm{A}$ or B1 [12]. In this study, all phylogenetic group B2 isolates were found in Cluster IV. The dependency between Cluster IV and phylogenetic group B2 was significant ( $X^{2}$ test, $P<0.05$ ), but no significance was found between other clusters and phylogenetic groups. In addition, it was always observed the number of virulence-associated genes in an isolate was significantly correlated with its phylogenetic group, thus B2 isolates contained more virulence-associated genes than non-B2 isolates $\left(\chi^{2}\right.$ test, $\left.P<0.05\right)$. The prevalence of virulence-associated genes between clusters was also compared (Table 2), but no characteristic pattern was found for ExPEC. However, the presence of $h l y D$, ibeA, usp, cnfl, papA in Cluster IV is a difference from the other three clusters, indicating that those five genes are possible markers for the prediction of highly virulent ExPEC.

\section{MLST analysis}

Among 21 isolates belonging to phylogenetic groups $\mathrm{D}$ and B2, 13 different STs were identified, including 11 known STs (ST1011, ST354, ST788, ST405, ST117, ST12, ST95, ST961, ST141, ST648 and ST457) (Additional file 1). Two novel STs were identified in this study, namely, ST5170 and ST5171. Four STs included all seven B2 group isolates, namely ST12, ST961, ST141, and ST95. Other STs were found for group D 
Table 1 Distribution of virulence factors classified according to phylogenetic group

\begin{tabular}{|c|c|c|c|c|c|}
\hline \multirow[t]{2}{*}{ Virulence factors } & \multicolumn{5}{|c|}{ NO. of ExPEC isolates(\%) } \\
\hline & Total $(n=64 ; \%)$ & Group A $(n=37 ; 57.8 \%)$ & GroupB1 $(n=6 ; 9.4 \%)$ & GroupB2 ( $(n=7 ; 10.9 \%)$ & Group D $(n=14 ; 21.9 \%)$ \\
\hline \multicolumn{6}{|l|}{ Siderophores } \\
\hline iutA & $39(60.1)$ & $24(64.9)^{a}$ & $1(16.7)^{\mathrm{b}}$ & $7(100)^{b}$ & $7(50)^{\mathrm{b}}$ \\
\hline $\operatorname{iroN}$ & $17(26.6)$ & $4(10.9)^{a}$ & $3(50)^{b}$ & $6(85.7)^{b}$ & $4(28.6)^{b}$ \\
\hline fyuA & 15(23.4) & $5(13.5)^{a}$ & $0(0)^{a}$ & $7(100)^{b}$ & $3(21.4)^{c}$ \\
\hline ireA & $7(10.9)$ & $3(8.1)$ & $0(0)$ & $1(14.3)$ & $3(21.4)$ \\
\hline \multicolumn{6}{|l|}{ Adhesins } \\
\hline $\mathrm{fimH}$ & $52(81.3)$ & $23(62.2)$ & $6(100)$ & $7(103)$ & 11(78.6) \\
\hline papC & $14(21.9)$ & $5(13.5)^{\mathrm{a}}$ & $1(16.7)^{\mathrm{ab}}$ & $5(71.4)^{b}$ & $3(21.4)^{b}$ \\
\hline papA & $3(4.7)$ & $0(0)^{a}$ & $0(0)^{\mathrm{ab}}$ & $3(42.9)^{b}$ & $0(0)^{b}$ \\
\hline$s f a A$ & $1(1.6)$ & $1(2.7)$ & $0(0)$ & $0(0)$ & $0(0)$ \\
\hline hraA & 13(20.3) & $4(10.9)^{a}$ & $0(0)^{a}$ & $5(71.4)^{\mathrm{b}}$ & $4(28.6)^{b}$ \\
\hline$a f a$ & $3(4.7)$ & $3(8.1)$ & $0(0)$ & $0(0)$ & $0(0)$ \\
\hline \multicolumn{6}{|l|}{ Toxin } \\
\hline cnfl & $5(7.8)$ & $0(0)^{a}$ & $0(0)^{a}$ & $5(71.4)^{b}$ & $0(0)^{c}$ \\
\hline hlyD & $5(7.8)$ & $0(0)^{a}$ & $0(0)^{a}$ & $5(71.4)^{\mathrm{b}}$ & $0(0)^{c}$ \\
\hline vat & $42(65.6)$ & $23(62.2)$ & $4(66.7)$ & $7(100)$ & $8(57.1)$ \\
\hline \multicolumn{6}{|l|}{ Miscellaneous } \\
\hline ompA & $64(100)$ & $37(100)$ & $6(100)$ & $7(100)$ & $14(100)$ \\
\hline ups & 7(10.9) & $0(0)^{a}$ & $0(0)^{a}$ & $7(100)^{b}$ & $0(0)^{c}$ \\
\hline trat & $40(62.5)$ & $30(81.1)^{\mathrm{a}}$ & $4(66.7)^{a b}$ & $3(42.9)^{b}$ & $3(21.4)^{b}$ \\
\hline \multicolumn{6}{|l|}{ Invasion } \\
\hline ibea & $2(3.1)$ & $0(0)^{a}$ & $0(0)^{\mathrm{ab}}$ & $2(28.6)^{b}$ & $0(0)^{b}$ \\
\hline \multicolumn{6}{|l|}{ Capsule } \\
\hline kpsMII & $10(15.7)$ & $0(0)^{a}$ & $0(0)^{a}$ & $7(100)^{b}$ & $3(21.4)^{c}$ \\
\hline kpsMIII & $1(1.6)$ & $0(0)$ & $0(0)$ & $0(0)$ & $1(7.1)$ \\
\hline
\end{tabular}

$\mathrm{a}, \mathrm{b}, \mathrm{c} P<0.05$

isolates. To further analyze the phylogenetic relationships of these 21 ExPEC isolates, a phylogenetic tree were generated (Fig. 2). The phylogenetic analysis showed that the group B2 and group D isolates could each be grouped (Fig. 2). All isolates in the group B2 section of the tree were classified into Cluster IV in the virulence genotyping analysis; the isolates in the group D part of the tree were from Clusters I, II and III (Fig. 2).

eBURST analysis of these 21 isolates identified nine clonal complex (CCs), namely, CC1011, CC457, CC117, CC141, CC405, CC354, CC648, CC12 and CC95 (Fig. 3). ST774 is a single locus variant (SLV) of ST648, which is the primary founder of CC648. ST961 was connected to ST12 as a member of CC12. Three STs were not classified into any CCs, i.e., they were singletons (ST5170, ST788, and ST5171). Strains from CC12 and CC95 have been indicated to have strong pathogenicity and zoonotic potential (Table 3).

\section{Virulence evaluation of representative ExPEC isolates}

Seven group B2 strains selected from Cluster IV showed high virulence in a mouse infection model (Table 4): the mortalities varied from $60 \%(3 / 5)$ to $100 \%(5 / 5)$ at a challenge dose of $4 \times 10^{7} \mathrm{CFU}$; the mortalities were more diverse at a challenge dose of $4 \times 10^{6} \mathrm{CFU}$, but of particular note, isolate JX131101 caused $100 \%$ mortality (5/5), indicating high virulence. The 14 group D strains showed obvious diversity in virulence: PxECZ01C and PxECZF4d did not cause any mortality at a dose of $4 \times 10^{7} \mathrm{CFU}$, while strains SXE-20 and SXE-14 presented high virulence; all of group $\mathrm{D}$ strains have no pathogenicity at a challenge dose of $4 \times 10^{6} \mathrm{CFU}$. No abnormality was found in mice in the control group injected with PBS.

\section{Discussion}

The importance of ExPEC has been recognized, and many ExPEC related diseases in cows, humans and food have been reported in the North America and 


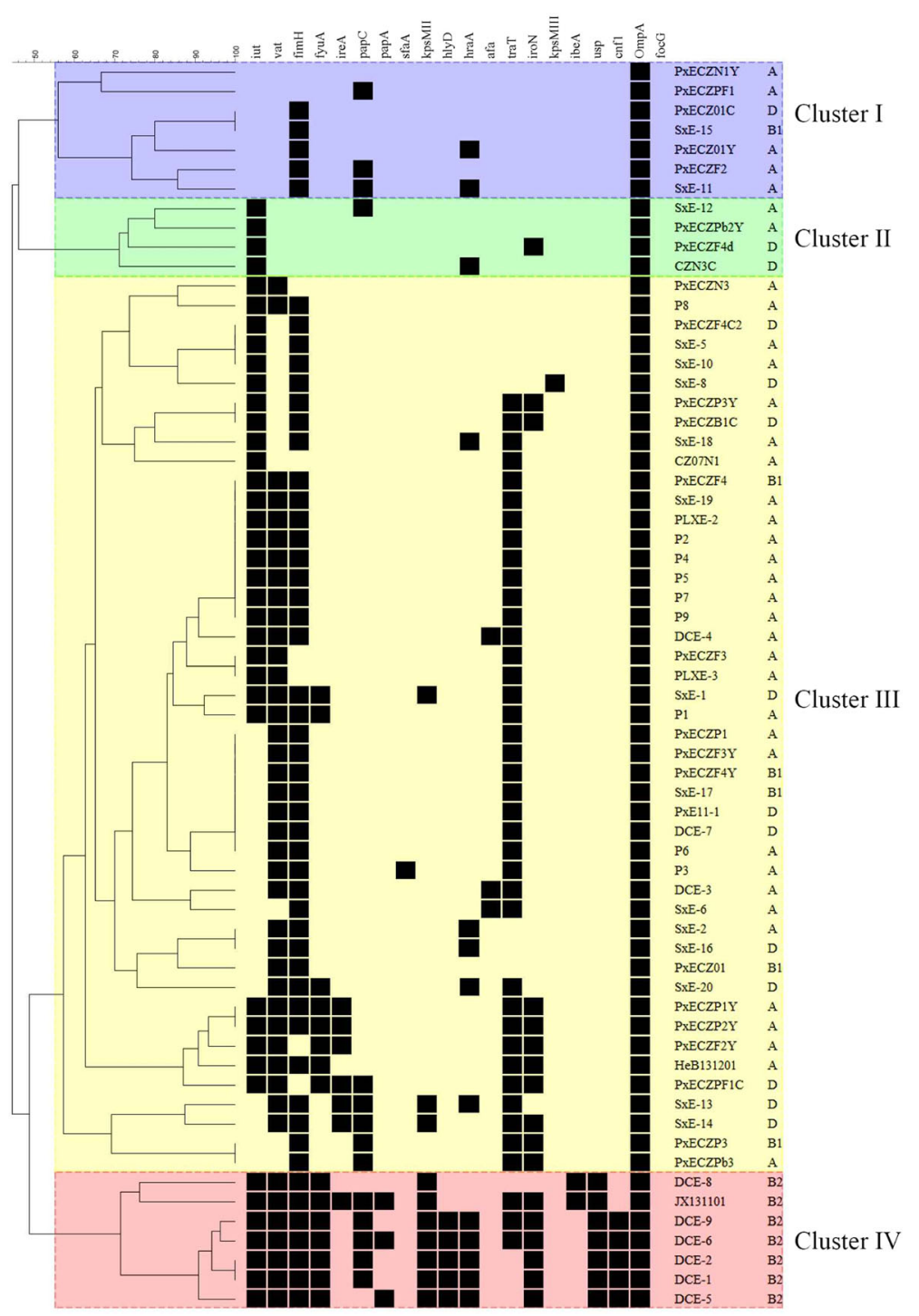

Fig. 1 Clustering of the 64 EXPEC isolates based on the presence or absence of virulence-associated factors. Each column shows the results for a single gene. Black: gene present; blank: gene absent

Europe. In China, ExPEC has become a frequent pathogen in the swine industry $[13,14]$. However, understanding of the pathogenesis and epidemiology of porcine ExPEC is still limited. Some reports showed that $>50 \%$ of ExPEC isolates from swine belong to phylogenetic groups A and B1 [15], which is consistent with our results. In this study, only a few isolates were classified as phylogenetic group B2, which is an obvious contrast with the fact that most ExPECs isolated from humans or companion animals belong to groups B2 and D [3]. In this study, the isolates were considered as ExPEC based organ origin. Johnson et al [16] proposed a molecular approach to delimit ExPEC, by considering the presence of 2 of the 5 virulence markers (papA/papC, sfa/foc, afa/dra, iutA and kpsMTII) in an E. coli strain as ExPEC. According to this criterion, 13 of 69 strains in this study were identified as ExPEC, which contained all 7 B2 strains (Additional file 3: Table S3). Notably, the prevalence of ExPEC that qualified to criterion was larger within high virulence strains $(9 / 12,75 \%$; mortality $\geq 3)$ than within low virulence strains $(2 / 9$, $22.2 \%$; mortality $\leq 2$ ) in the mouse infection model. This indicated that Johnson's criterion is more strict and selective compared to the robust standard we used in this study, and is able to identify strains with a higher potential to cause diseases.

A single virulence factor does not determine bacterial pathogenicity. Thus, in this study, 19 major virulence-associated genes of ExPEC were selected 
Table 2 Distribution of virulence factors classified according to clusters

\begin{tabular}{|c|c|c|c|c|c|}
\hline \multirow{2}{*}{$\begin{array}{l}\text { Virulence } \\
\text { factors }\end{array}$} & \multicolumn{5}{|c|}{ NO. of ExPEC isolates(\%) } \\
\hline & Total $(n=64 ; \%)$ & Cluster I $(n=7 ; 10.9 \%)$ & $\begin{array}{l}\text { Cluster II } \\
(n=4 ; 6.25 \%)\end{array}$ & $\begin{array}{l}\text { Cluster III } \\
(n=46 ; 71.9 \%)\end{array}$ & $\begin{array}{l}\text { Cluster IV } \\
(n=7 ; 10.9 \%)\end{array}$ \\
\hline \multicolumn{6}{|c|}{ Siderophores } \\
\hline iutA & $39(60.1)$ & $0(0)^{a}$ & $4(100)^{b}$ & $28(60.1)^{b}$ & $7(100)^{b}$ \\
\hline iroN & 17(26.6) & $0(0)^{\mathrm{a}}$ & $1(25)^{\mathrm{ab}}$ & $10(21.7)^{a}$ & $6(85.7)^{\mathrm{b}}$ \\
\hline fyuA & 15(23.4) & $0(0)^{a}$ & $0(0)^{\mathrm{a}}$ & $8(17.4)^{a}$ & $7(100)^{b}$ \\
\hline ireA & $7(10.9)$ & $0(0)$ & $0(0)$ & $6(13)$ & $1(14.3)$ \\
\hline \multicolumn{6}{|l|}{ Adhesins } \\
\hline $\mathrm{fimH}$ & $52(81.3)$ & $5(71.4)^{\mathrm{ac}}$ & $0(0)^{b}$ & $40(86.9)^{c}$ & $7(100)^{c}$ \\
\hline papC & 14(21.9) & $3(42.9)$ & $1(25)$ & $5(10.9)$ & $5(71,4)$ \\
\hline papA & $3(4.7)$ & $0(0)$ & $0(0)$ & $0(0)$ & $3(42.9)$ \\
\hline$s f a A$ & $1(1.6)$ & $0(0)$ & $0(0)$ & $1(2.2)$ & $0(0)$ \\
\hline hraA & 13(20.3) & $2(28.6)$ & $1(25)$ & $5(10.9)$ & $5(71.4)$ \\
\hline$a f a$ & $3(4.7)$ & $0(0)$ & $0(0)$ & $3(6.5)$ & $0(0)$ \\
\hline \multicolumn{6}{|l|}{ Toxin } \\
\hline cnfl & $5(7.8)$ & $0(0)^{a}$ & $0(0)^{a}$ & $0(0)^{a}$ & $5(71.4)^{b}$ \\
\hline hlyD & $5(7.8)$ & $0(0)^{a}$ & $0(0)^{\mathrm{a}}$ & $0(0)^{a}$ & $5(71.4)^{b}$ \\
\hline vat & $42(65.6)$ & $0(0)^{a}$ & $0(0)^{a}$ & $35(76.1)^{b}$ & $7(100)^{b}$ \\
\hline \multicolumn{6}{|c|}{ Miscellaneous } \\
\hline ompA & $64(100)$ & $7(100)$ & $4(100)$ & $46(100)$ & $7(100)$ \\
\hline ups & $7(10.9)$ & $0(0)^{a}$ & $O(0)^{a}$ & $0(0)^{\mathrm{a}}$ & $7(100)^{b}$ \\
\hline trat & $40(62.5)$ & $0(0)^{a}$ & $0(0)^{a}$ & $37(80.4)^{b}$ & $3(42.9)^{b}$ \\
\hline \multicolumn{6}{|l|}{ Invasion } \\
\hline ibeA & $2(3.1)$ & $0(0)$ & $0(0)$ & $0(0)$ & $2(28.6)$ \\
\hline \multicolumn{6}{|l|}{ Capsule } \\
\hline kpsMIII & $1(1.6)$ & $0(0)$ & $0(0)$ & $1(2.2)$ & $0(0)$ \\
\hline kpsMII & 10(15.7) & $0(0)^{\mathrm{a}}$ & $0(0)^{a}$ & $3(6.5)^{\mathrm{a}}$ & $7(100)^{b}$ \\
\hline
\end{tabular}

$\mathrm{a}, \mathrm{b}, \mathrm{c} P<0.05$

for a comprehensive analysis with phylogenetic investigation, aiming to reveal genotypic traits related to virulence. These 19 virulence-associated genes contribute to the fitness of ExPEC and increase its adaptability and competitiveness in colonizing host animals. Four clusters were identified in the genotyping analysis. Cluster IV included only B2 group isolates and showed a higher prevalence of virulenceassociated genes than the other three clusters. Animal infection experiments confirmed higher virulence of isolates from Cluster IV, which is consistent with B2 group strains leading to serious diseases [17]. The distinction between B2 and non-B2 ExPEC is clear, shown by MLST, virulence genotyping and virulence assessment. In addition, group D ExPEC isolates are considered to be more pathogenic than groups $\mathrm{A}$ and B1, although in this study some group D strains presented similar virulence to group A or B1 isolates.
Furthermore, group D isolates were mixed with group A and B1 isolates in Clusters I, II and III in the genotyping analysis. In MLST analysis, no obvious correlations were observed with genotypic cluster virulence in group D strains. Group D isolates thus present high diversity in genotype and phenotype.

MLST analysis can provide other information besides phylogenetic relationships [18]. After comparing our results with the MLST database (http://pubmlst.org/), we found STs in this study that have been reported to be present in diseases of birds, dogs and humans. For example, strain JX131101 belonged to ST95 in CC95; ST95 is the most important pathogenic sequence type in human-associated cases [19]. ST95 isolates are frequently reported to be related to newborn meningitis (NM) and uropathogenic infection (UTI) in Europe and the North America [20, 21]. Group D isolate CZN3C is assigned to ST354, which 


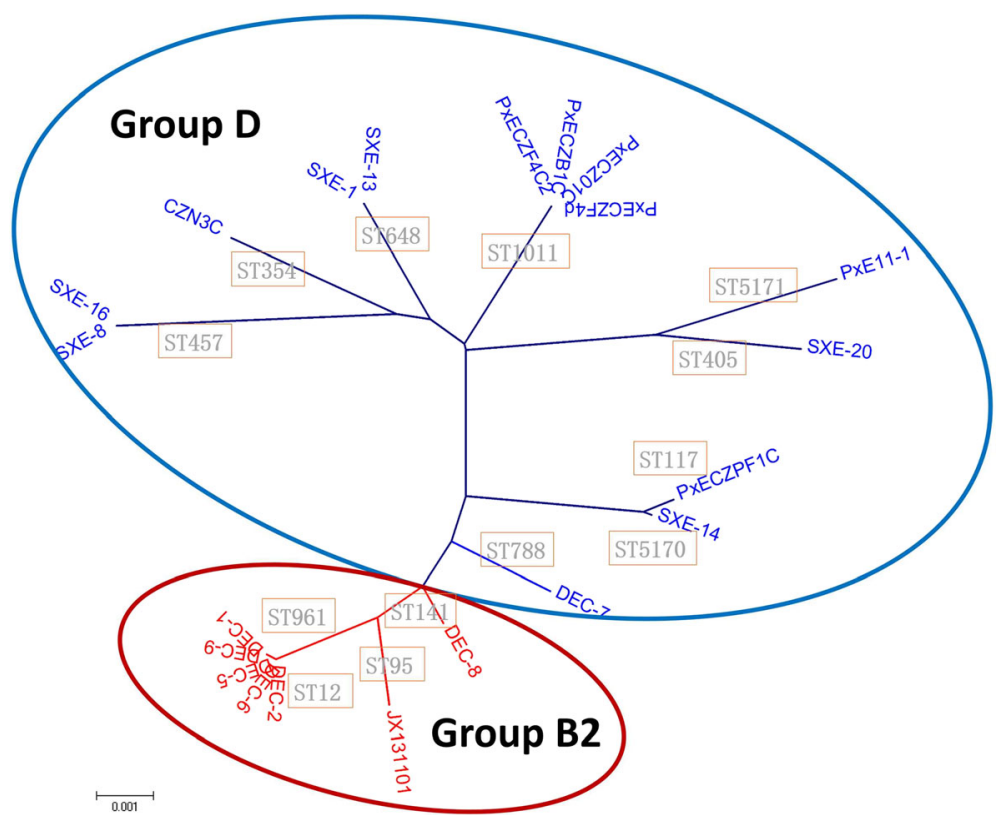

Fig. 2 Phylogenetic organization of group B2 and D isolates of different multi locus sequence types based on the neighbor-joining method. Numbers at the nodes are bootstrap values and the bar represents 0.001 nucleotide changes. Group B2 strains gather together (red circle), as do group D strains (blue circle)

is a common ST type in isolates causing UTI and $\mathrm{NM}$ in humans, companion animals and swine $[22,23]$. Some ST1011 isolates lead to UTI in avians and companion animals [24], but several group D ST1011 isolates showed low virulence in this study. Our data suggest that isolates from the same ST can have different pathogenic phenotypes, and indicate the diversity and complexity of pathogenic E. coli.

A study by Tan et al. showed that fimH, traT and iutA were highly prevalent in porcine ExPEC isolates, which is in consistent with our results, but the prevalence of fyuA, cnf1, kpsMTII, and iroN differ between our study and theirs; the foc $G$ gene was not detected in any isolates in this study, which is similar to Tan's research [25]. Interestingly, the results of VR gene detection in ExPEC isolates from retail pork is quite different from that from pigs on farms [26]. This suggests that E. coli strains isolated from pork may originate from processes such as meat production or transportation.

We found five VR genes to be highly prevalent specifically in Cluster IV (which contained only group B2 isolates), namely $p a p A, h l y D$, usp, ibeA and cnf1, suggesting that these five genes may be closely related to strong pathogenicity. This result contains a risk factor that similar geographical of B2 isolated in this study might lead to a prevalence bias of virulence factors. Both $h l y D$ and cnfl are well-known toxin genes in
ExPEC. ibeA is associated with neonatal meningitis [27] and is an important virulence-associated factor widely distributed among APEC and NMEC [28]. usp, the uropathogenic-specific gene, was reported in a large number of strains from patients and animals with pyelonephritis or prostatitis [29]. Both our results and a previous study of virulence genotypes of canine ExPEC [11] indicate that cnfl, hlyD, ups and kpsMII could be possible marker genes for high virulence in ExPEC, although this point still needs to be verified in further study or using a larger collection of ExPEC isolates.

\section{Conclusions}

In this study, a total of 19 VR genes in 64 Chinese porcine ExPEC strains were detected by PCR. Based on VR gene profiles, those isolates were divided into four clusters (named I to IV). Notably, all Cluster IV isolates were from group B2, and VR genes were remarkably more prevalent in group B2 than in groups $\mathrm{A}, \mathrm{B} 1$ and $\mathrm{D}$. The distinction between group $\mathrm{B} 2$ and non-B2 isolates in virulence and genotype accorded with MLST analysis. Considering Cluster IV isolates had the strongest pathogenicity in a mouse infection model, genes that were only found in group B2 strains (that cnf1, hlyD, ups and kpsMII) could be possible marker genes for high virulence in ExPEC. This study revealed the genetic differences between 


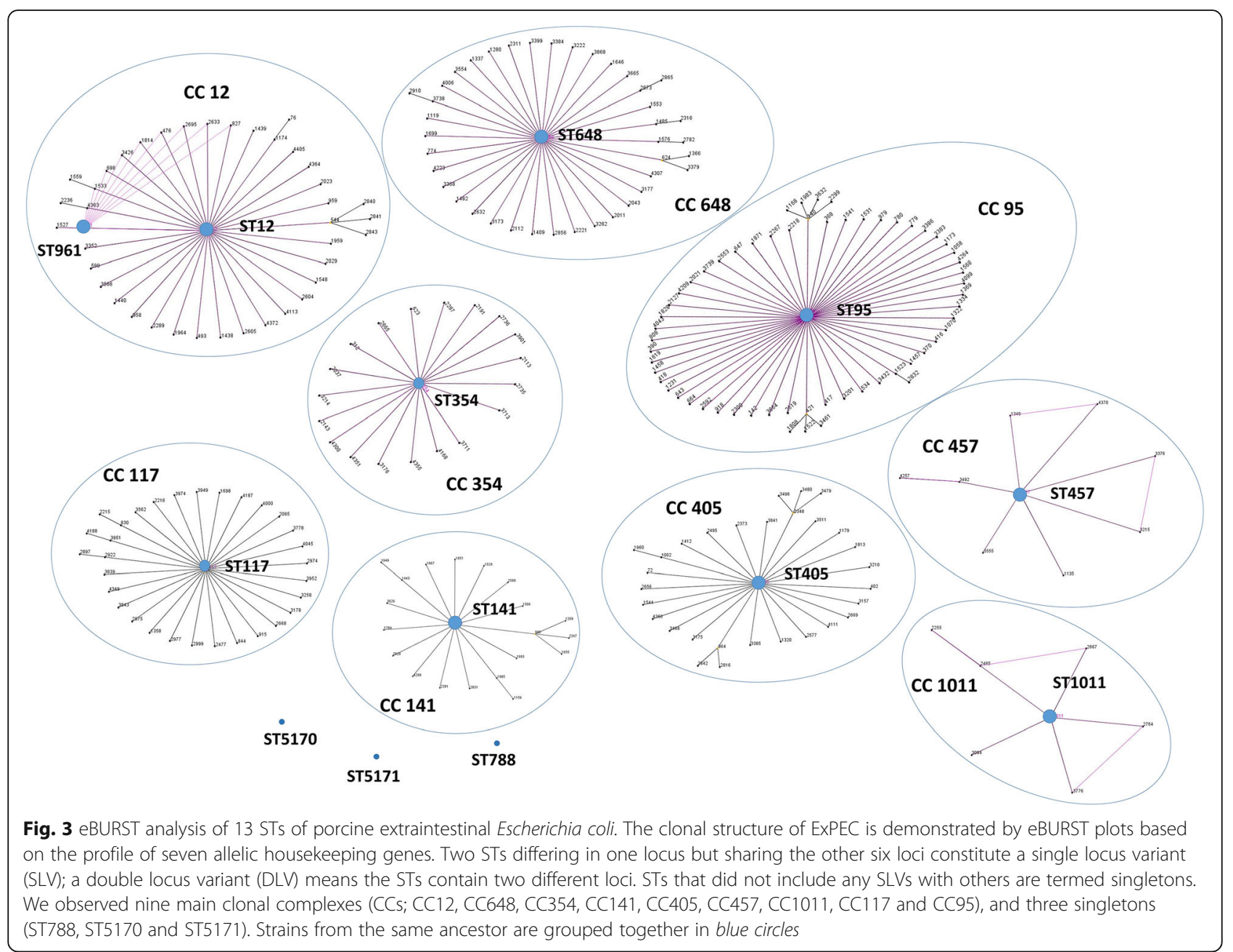

porcine ExPEC strains from China and enlarges the knowledge of the porcine ExPEC virulence.

\section{Methods}

Bacterial isolation and culture conditions

From April 2013 to August 2014, a total of 64 ExPEC strains were isolated from clinically diseased pigs in five provinces (Jiangsu, Zhejiang, Shandong, Hebei, Shanxi and Jiangxi) of China (Additional file 4: Table S2). All the isolates were recovered from extraintestinal organs, including spleen, lung, liver, kidney and brain, and MacConkey agar was used for initial purification. Isolates were grown in Luria-Bertani broth for $6 \mathrm{~h}$ and then stored in $50 \%$ glycerol at $-70{ }^{\circ} \mathrm{C}$ until further characterization.

\section{Hemolytic activity tests}

The hemolytic activities of clinical ExPEC isolates were determined by culture on $7 \%$ sheep blood agar. The formation of beta-hemolysin was observed.
PCR detection of virulence-related genes and phylogenetic group identification

The genomic DNA of bacteria was extracted using an E.Z.N.A. bacterial DNA kit (Omega, Beijing, China) following the manufacturer's instructions. Individual PCR assays were performed for 19 ExPEC VR genes, including kpsMII, kpsMIII, iroN, fyuA, ireA, iutA, papC, hra, sfaS, papA, fimH, afa, hlyD, vat, cnfl, ompA, traT, usp and ibeA. The phylogenetic group was determined by multiplex PCR with three pairs of primers, chuA, yjaA and TspE4.C2 [30]. All PCR primers used in this study are listed in Table 5.

\section{Cluster analysis of virulence genotype}

The presence or absence of putative virulenceassociated genes among isolates was visualized using BioNumerics (version 6.6, Applied Maths, Kortrijk, Belgium) to study similarity among isolates, according to the method applied in a previous report [11]. These data were analyzed using a simple matching coefficient. Cluster analysis was performed by the 
Table 3 Sequence types and clonal complex of group D and B2 isolates

\begin{tabular}{|c|c|c|c|}
\hline Sequence type (ST) & Strains & Clusters & Clonal complex \\
\hline ST1011 & PxECZ01C & Cluster I & CC1011 \\
\hline ST1011 & PxECZF4d & Cluster II & CC1011 \\
\hline ST354 & CZN3C & Cluster II & CC354 \\
\hline ST1011 & PXECZB1C & Cluster III & CC1011 \\
\hline ST1011 & PxECZF4C2 & Cluster III & CC1011 \\
\hline ST457 & SXE-8 & Cluster III & CC457 \\
\hline \multirow{2}{*}{$\begin{array}{l}\text { ST648 } \\
\text { ST648 }\end{array}$} & SXE-1 & Cluster III & CC648 \\
\hline & SXE-13 & Cluster III & CC648 \\
\hline ST5171 & PXE11-1 & Cluster III & None \\
\hline ST788 & DCE-7 & Cluster III & None \\
\hline ST457 & SXE-16 & Cluster III & CC457 \\
\hline ST405 & SXE-20 & Cluster III & CC405 \\
\hline ST117 & PxECZPF1C & Cluster III & CC117 \\
\hline ST5170 & SXE-14 & Cluster III & None \\
\hline \multirow{2}{*}{$\begin{array}{l}\text { ST961 } \\
\text { ST961 }\end{array}$} & DCE-9 & Cluster IV & CC12 \\
\hline & DCE-1 & Cluster IV & $\mathrm{CC} 12$ \\
\hline ST12 & DCE-6 & Cluster IV & $\mathrm{CC} 12$ \\
\hline ST12 & DCE-2 & Cluster IV & CC12 \\
\hline ST12 & DCE-5 & Cluster IV & CC12 \\
\hline ST141 & DCE-8 & Cluster IV & CC141 \\
\hline ST95 & JX131101 & Cluster IV & CC95 \\
\hline
\end{tabular}

unweighted pair group method using arithmetic averages (UPGMA).

\section{Multilocus sequence typing}

MLST is a useful method to track genetic variation of microbes and survey epidemics. It provides an accurate and highly discriminatory genotyping system for bacteria. In this study, group D and group B2 ExPEC isolates were used to perform MLST tests. The DNA fragments of seven housekeeping genes (purA, fum $C$, $i c d, m d h, r e c A, \operatorname{gyr} B$ and $a d k$ ) were amplified by PCR and the products were sequenced. Allele sequence determination was performed using the E. coli MLST database (http://mlst.ucc.ie/mlst/dbs/E. coli). Any new alleles (i.e. those not recognized by the E. coli MLST system) were submitted to the database curator to be assigned new allele numbers. Sequence types (STs) were identified based on the allele profiles.

Phylogenetic analyses were conducted on the basis of the concatenation of seven MLST loci (purA, fum $C$, icd, $m d h$, recA, gyrB and $a d k)$ using the MEGA v4.1 software as previously described [31]. The result is presented as a neighbour-joining tree; the robustness of the groupings was assessed by bootstrap
Table 4 Experimental virulence of representative isolates in $\mathrm{BALB} / \mathrm{c}$ mice

\begin{tabular}{|c|c|c|c|c|c|}
\hline \multirow[t]{2}{*}{ Clusters } & \multirow[t]{2}{*}{ Strains } & \multicolumn{2}{|c|}{ Mortality } & \multirow{2}{*}{$\begin{array}{l}\text { Phylogenetic } \\
\text { groups }\end{array}$} & \multirow{2}{*}{ Virulence } \\
\hline & & $4 \times 10^{7}$ & $4 \times 10^{6}$ & & \\
\hline Cluster I & PxECZ01C & $0 / 5$ & $0 / 5$ & D & None virulence \\
\hline Cluster II & PxECZF4d & $0 / 5$ & $0 / 5$ & D & None virulence \\
\hline Cluster II & CZN3C & $2 / 5$ & $0 / 5$ & D & Less virulence \\
\hline Cluster III & PXECZF4C2 & $1 / 5$ & $0 / 5$ & D & Less virulence \\
\hline Cluster III & SXE-8 & $3 / 5$ & $0 / 5$ & D & Less virulence \\
\hline Cluster III & PXECZB1C & $2 / 5$ & $0 / 5$ & D & Less virulence \\
\hline Cluster III & SXE-1 & $2 / 5$ & $0 / 5$ & D & Less virulence \\
\hline Cluster III & PxE11-1 & $2 / 5$ & $0 / 5$ & D & Less virulence \\
\hline Cluster III & DCE-7 & $1 / 5$ & $0 / 5$ & $\mathrm{D}$ & Less virulence \\
\hline Cluster III & SXE-16 & $3 / 5$ & $0 / 5$ & D & Less virulence \\
\hline Cluster III & SXE-20 & $4 / 5$ & $0 / 5$ & D & Less virulence \\
\hline Cluster III & PxECZPF1C & $3 / 5$ & $0 / 5$ & $D$ & Less virulence \\
\hline Cluster III & SXE-13 & $1 / 5$ & $0 / 5$ & D & Less virulence \\
\hline Cluster III & SXE-14 & $5 / 5$ & $1 / 5$ & D & Virulence \\
\hline Cluster IV & DCE-9 & $3 / 5$ & $0 / 5$ & B2 & Less virulence \\
\hline Cluster IV & DCE-6 & $4 / 5$ & $1 / 5$ & B2 & Virulence \\
\hline Cluster IV & DCE-2 & $3 / 5$ & $0 / 5$ & B2 & Less virulence \\
\hline Cluster IV & DCE-5 & $4 / 5$ & $3 / 5$ & B2 & Virulence \\
\hline Cluster IV & DCE-1 & $4 / 5$ & $2 / 5$ & B2 & Virulence \\
\hline Cluster IV & DCE-8 & $4 / 5$ & $2 / 5$ & B2 & Virulence \\
\hline \multirow[t]{2}{*}{ Cluster IV } & JX131101 & $5 / 5$ & $5 / 5$ & B2 & Virulence \\
\hline & PBS & \multicolumn{4}{|c|}{ No mortality } \\
\hline
\end{tabular}

All these strains were able to kill BALB/c mice in doses of $10^{8} \mathrm{cfu}$, including PxECZ01C and PxECZF4d

resampling of 1,000 replicates. eBURST (http:// eburst.mlst.net) analysis was also performed to identify potential clonal complexes (CCs) and founders [18], and the overall population structures were determined.

\section{Statistical analysis}

The prevalence of VR genes in ExPEC isolates in different clusters was compared using Fisher's exact test (Statistica 5.0, StatSoft Inc., Tulsa, OK, USA). A chisquare test $\left(\chi^{2}\right.$ test) was used to calculate the correlation between clusters, phylogenetic groups and the quantity of virulence-associated genes. Results were considered significant if $P<0.05$.

\section{Animal infection experiments}

E. coli strains belonging to groups D and B2 have a higher risk of causing severe disease. Using the results of genotype clustering, 21 group D and B2 ExPEC strains were tested in a mouse infection model. All 
Table 5 Virulence genes primers for PCR in this study

\begin{tabular}{|c|c|c|c|}
\hline PCR assay & Gene & Primer sequence & $\begin{array}{l}\text { PCR product } \\
\text { size/bp }\end{array}$ \\
\hline \multirow{38}{*}{$\begin{array}{l}\text { Virulence- } \\
\text { associated } \\
\text { genes }\end{array}$} & \multirow[t]{2}{*}{ papA } & ATGGCAGTGGTGTCTTTTGGTG & \multirow[t]{2}{*}{717} \\
\hline & & CGTCCCACCATACGTGCTCTTC & \\
\hline & \multirow[t]{2}{*}{ papC } & GTGGCAGTATGAGTAATGACCGTTA & \multirow[t]{2}{*}{203} \\
\hline & & ATATCCTTTCTGCAGGGATGCAATA & \\
\hline & \multirow[t]{2}{*}{ iutA } & ATCGGCTGGACATCATGGGAAC & \multirow[t]{2}{*}{314} \\
\hline & & CGCATTTACCGTCGGGAACGG & \\
\hline & \multirow[t]{2}{*}{ vat } & CTTACCTCTCTGGCACTATCTG & \multirow[t]{2}{*}{1021} \\
\hline & & GTCAGTGAACCGGCACC & \\
\hline & \multirow[t]{2}{*}{$\mathrm{fimH}$} & TGCAGAACGGATAAGCCGTGG & \multirow[t]{2}{*}{508} \\
\hline & & GCAGTCACCTGCCCTCCGGTA & \\
\hline & \multirow[t]{2}{*}{ fyuA } & TGATTAACCCCGCGACGGGAA & \multirow[t]{2}{*}{787} \\
\hline & & CGCAGTAGGCACGATGTTGTA & \\
\hline & \multirow[t]{2}{*}{ ireA } & GATGACTCAGCCACGGGTAA & \multirow[t]{2}{*}{254} \\
\hline & & CCAGGACTCACCTCACGAAT & \\
\hline & \multirow[t]{2}{*}{ kpsMTII } & GCGCATTTGCTGATACTGTTG & \multirow[t]{2}{*}{272} \\
\hline & & CATCCAGACGATAAGCATGAGCA & \\
\hline & \multirow[t]{2}{*}{ hlyD } & CTCCGGTACGTGAAAAGGAC & \multirow[t]{2}{*}{904} \\
\hline & & GCCCTGATTACTGAAGCCTG & \\
\hline & \multirow[t]{2}{*}{ sfas } & GTCTCTCACCGGATGCCAGAATAT & \multirow[t]{2}{*}{240} \\
\hline & & GCATTACTTCCATCCCTGTCCTG & \\
\hline & \multirow[t]{2}{*}{ hra } & GTAACTCACACTGCTGTCACCT & \multirow[t]{2}{*}{139} \\
\hline & & CGAATCGTTGTCACGTTCAG & \\
\hline & \multirow[t]{2}{*}{ cnf1 } & CTITACAATATTGACATGCTG & \multirow[t]{2}{*}{446} \\
\hline & & TCGTTATAAAATCAAACAGTG & \\
\hline & \multirow[t]{2}{*}{$a f a$} & TAAGGAAGTGAAGGAGCGTG & \multirow[t]{2}{*}{210} \\
\hline & & CCGCCCTGAAGAAGTATCAC & \\
\hline & \multirow[t]{2}{*}{ kpsMTIII } & TCCTCTTGCTACTATTCССCCT & 392 \\
\hline & & AGGCGTATCCATCCCTCCTAAC & \\
\hline & $i b e A$ & AATGAGTGCCGCTCGTGAAGG & 548 \\
\hline & & CCCCCCAGTCTCCATATTTAG & \\
\hline & $\operatorname{iroN}$ & CTCCGACGATGATAATGACGA & 440 \\
\hline & & TGGGACGTITGGTAATGATGT & \\
\hline & ompA & GATCAGTGCAGCACGCTGTTT & 385 \\
\hline & & GGGCGAAGCAGCTCCAGTAG & \\
\hline & trat & CGATGAGCACAGCAATCAAGA & 486 \\
\hline & & CATTATCCGTTGTCACCGTTG & \\
\hline & usp & GAGCGGTTATTTATTGAAATC & 448 \\
\hline & & CACAGCAGTCATCAACCACTG & \\
\hline
\end{tabular}

isolates tested were grown in LB medium with shaking at $37{ }^{\circ} \mathrm{C}$ for about $4-5 \mathrm{~h}$ to reach a concentration of $10^{9} \mathrm{CFU} / \mathrm{ml}$.

A total of 215 female BALB/c mice (5-weeks-old) were purchased from the Comparative Medicine Center of
Yangzhou University. Mice were housed in the Animal Experimental Center of Nanjing Agricultural University, by a full-time staff. Temperature and humidity are controlled, food and water are accessible. The mice were divided into 43 groups, five mice per group. Mice in groups 1 to 42 were inoculated with the 21 selected strains by intraperitoneal injection $(0.2 \mathrm{~mL})$ at doses of $4 \times 10^{6}$ or $4 \times 10^{7} \mathrm{CFU}$ per mouse. Group 43 was the uninoculated control and received phosphate buffered saline (PBS). The mice were monitored for survival twice a day for 1 week.

\section{Additional files}

\section{Additional file 1: MLST and VR genes profiles. (XLSX $16 \mathrm{~kb}$ )}

Additional file 2: Table S1. Statistical analysis of associations between virulence-associated factores(VFs). $P$ values by Fisher's exact test, shown only where $<.10 . P<.10$ reflects statistical significance $; P$ values between .01 and .05 reflect possible statistical significance. (DOCX 19 kb)

Additional file 3: Table S2. The isolation sources of strains in this study. (DOCX $18 \mathrm{~kb}$ )

Additional file 4: Table S3. The information of isolates identified as EXPEC with Johnson et al's criterion (DOCX $15 \mathrm{~kb}$ )

\section{Abbreviations}

ExPEC: Extraintestinal pathogenic Escherichia coli; MLST: Multilocus sequence typing; VR: Virulence-related

\section{Acknowledgements}

We would like to thank Dr. Xian Qi and Dr. Chen Dong (Jiangsu Provincial Center for Disease Control and Prevention) for their help in using BioNumerics software.

\section{Funding}

This work was supported by grants from the National Nature Science Foundation of China (No. 31372455) and the project funded by the Priority Academic Program Development of Jiangsu Higher Education Institutions (PAPD).

Availability of data and materials

All data are fully available without restriction and is contained within the manuscript and supplemental materials.

\section{Authors' contributions}

HY conceived and designed the experiments. YZ, WD, LY, JM and MA performed the experiments. GL contributed materials and analysis tools. $Y Z$, $J M$, and ZP analyzed the data. YZ and WD drafted the manuscript. ZP and $\mathrm{HY}$ revised the manuscript. HY coordinated the study. All authors have read and approved the final manuscript version.

\section{Competing interests}

The authors declare that they have no competing interests.

\section{Ethics approval}

All animal experiments in this study were approved by the by the Animal Care and Use Committee of Nanjing Agricultural University, license number SYXK (SU) 2011-0036. All efforts were made to minimize suffering.

Received: 30 September 2016 Accepted: 15 February 2017 Published online: 08 April 2017

\section{References}

1. Johnson JR, Johnston B, Clabots CR, Kuskowski MA, Roberts E, DebRoy C. Virulence genotypes and phylogenetic background of Escherichia coli serogroup $\mathrm{O} 6$ isolates from humans, dogs, and cats. J Clin Microbiol. 2008;46(2):417-22 
2. Dezfulian H, Batisson I, Fairbrother JM, Lau PCK, Nassar A, Szatmari G, Harel J. Presence and characterization of extraintestinal pathogenic Escherichia coli virulence genes in F165-positive E-coli strains isolated from diseased calves and pigs. J Clin Microbiol. 2003;41(4):1375-85.

3. Maynard C, Bekal S, Sanschagrin F, Levesque RC, Brousseau R, Masson L, Lariviere S, Harel J. Heterogeneity among virulence and antimicrobial resistance gene profiles of extraintestinal Escherichia coli isolates of animal and human origin. J Clin Microbiol. 2004;42(12):5444-52.

4. Kohler CD, Dobrindt U. What defines extraintestinal pathogenic Escherichia coli? Int J Med Microbiol. 2011;301(8):642-7.

5. Picard B, Garcia JS, Gouriou S, Duriez P, Brahimi N, Bingen E, Elion J, Denamur $E$. The link between phylogeny and virulence in Escherichia coli extraintestinal infection. Infect Immun. 1999;67(2):546-53.

6. Russo TA, Johnson JR. Medical and economic impact of extraintestinal infections due to Escherichia coli: focus on an increasingly important endemic problem. Microbes Infect. 2003;5(5):449-56.

7. Normile D. Infectious diseases - WHO probes deadliness of China's pig-borne disease. Science. 2005;309(5739):1308-9.

8. Moulin-Schouleur M, Schouler C, Tailliez P, Kao MR, Bree A, Germon P, Oswald E, Mainil J, Blanco M, Blanco J. Common virulence factors and genetic relationships between 018: K1: H7 Escherichia coli isolates of human and avian origin. J Clin Microbiol. 2006;44(10):3484-92.

9. Boerlin P, Travis R, Gyles CL, Reid-Smith R, Janecko N, Lim H, Nicholson V, McEwen SA, Friendship R, Archambault M. Antimicrobial resistance and virulence genes of Escherichia coli isolates from swine in Ontario. Appl Environ Microbiol. 2005;71(11):6753-61.

10. Johnson JR, Delavari P, Kuskowski M, Stell AL. Phylogenetic distribution of extraintestinal virulence-associated traits in Escherichia coli. J Infect Dis. 2001;183(1):78-88.

11. Mateus L, Henriques S, Merino C, Pomba C, da Costa LL, Silva E. Virulence genotypes of Escherichia coli canine isolates from pyometra, cystitis and fecal origin. Vet Microbiol. 2013;166(3-4):590-4.

12. Moreno E, Prats G, Planells I, Planes AM, Perez T, Andreu A. Characterization of Escherichia coli isolates derived from phylogenetic groups A and B1 causing extraintestinal infection. Enferm Infecc Microbiol Clin. 2006;24(8):483-9.

13. Tang X, Tan C, Zhang X, Zhao Z, Xia X, Wu B, Guo A, Zhou R, Chen H. Antimicrobial resistances of extraintestinal pathogenic Escherichia coli isolates from swine in China. Microb Pathog. 2011;50(5):207-12.

14. Liu C, Chen Z, Tan C, Liu W, Xu Z, Zhou R, Chen H. Immunogenic characterization of outer membrane porins OmpC and OmpF of porcine extraintestinal pathogenic Escherichia coli. FEMS Microbiol Lett. 2012;337(2):104-11.

15. Ding Y, Tang X, Lu P, Wu B, Xu Z, Liu W, et al. Clonal analysis and virulent traits of pathogenic extraintestinal Escherichia coli isolates from swine in China. BMC veterinary research. 2012;8:140.

16. Johnson JR, Murray AC, Gajewski A, Sullivan M, Snippes P, Kuskowski MA, Smith KE. Isolation and molecular characterization of nalidixic acid-resistant extraintestinal pathogenic Escherichia coli from retail chicken products. Antimicrob Agents Chemother. 2003;47(7):2161-8

17. Vangchhia B, Abraham S, Bell JM, Collignon P, Gibson JS, Ingram PR, et al. Phylogenetic diversity, antimicrobial susceptibility and virulence characteristics of phylogroup F Escherichia coli in Australia. Microbiol. 2016;162(11):1904-12.

18. Feil EJ, Li BC, Aanensen DM, Hanage WP, Spratt BG. eBURST: Inferring patterns of evolutionary descent among clusters of related bacterial genotypes from multilocus sequence typing data. J Bacteriol. 2004; 186(5):1518-30

19. Tivendale KA, Logue CM, Kariyawasam S, Jordan D, Hussein A, Li G, Wannemuehler Y, Nolan LK. Avian-pathogenic Escherichia coli strains are similar to neonatal meningitis $E$. coli strains and are able to cause meningitis in the rat model of human disease. Infect Immun. 2010; 78(8):3412-9.

20. Moriel DG, Bertoldi I, Spagnuolo A, Marchi S, Rosini R, Nesta B, Pastorello I, Corea VA, Torricelli G, Cartocci E, et al. Identification of protective and broadly conserved vaccine antigens from the genome of extraintestinal pathogenic Escherichia coli. Proc Natl Acad Sci U S A. 2010;107(20):9072-7.

21. Bauchart $\mathrm{P}$, Germon P, Bree A, Oswald E, Hacker J, Dobrindt U. Pathogenomic comparison of human extraintestinal and avian pathogenic Escherichia coli-search for factors involved in host specificity or zoonotic potential. Microb Pathog. 2010;49(3):105-15.

22. Guo S, Wakeham D, Brouwers HJ, Cobbold RN, Abraham S, Mollinger JL, Johnson JR, Chapman TA, Gordon DM, Barrs VR, et al. Human-associated fluoroquinolone-resistant Escherichia coli clonal lineages, including ST354, isolated from canine feces and extraintestinal infections in Australia. Microbes Infect / Institut Pasteur. 2015;17(4):266-74.

23. Mora A, Blanco M, Lopez C, Mamani R, Blanco JE, Alonso MP, Garcia-Garrote F, Dahbi G, Herrera A, Fernandez A, et al. Emergence of clonal groups O1: HNM-D-ST59, O15:H1-D-ST393, O20:H34/HNM-D-ST354, O25b:H4-B2-ST131 and ONT:H21,42-B1-ST101 among CTX-M-14-producing Escherichia col clinical isolates in Galicia, northwest Spain. Int J Antimicrob Agents. 2011;37(1):16-21

24. Maluta RP, Logue CM, Casas MR, Meng T, Guastalli EA, Rojas TC, et al. Overlapped sequence types (STs) and serogroups of avian pathogenic (APEC) and human extra-intestinal pathogenic (ExPEC) Escherichia coli isolated in Brazil. Plos One. 2014;9(8):e105016.

25. Tan C, Tang X, Zhang X, Ding Y, Zhao Z, Wu B, Cai X, Liu Z, He Q, Chen H. Serotypes and virulence genes of extraintestinal pathogenic Escherichia coli isolates from diseased pigs in China. Vet J. 2012;192(3):483-8.

26. Xia X, Meng J, Zhao S, Bodeis-Jones S, Gaines SA, Ayers SL, McDermott PF. Identification and antimicrobial resistance of extraintestinal pathogenic Escherichia coli from retail meats. J Food Prot. 2011;74(1):38-44.

27. Bingen E, Picard B, Brahimi N, Mathy S, Desjardins P, Elion J, Denamur E. Phylogenetic analysis of Escherichia coli strains causing neonatal meningitis suggests horizontal gene transfer from a predominant pool of highly virulent B2 group strains. J Infect Dis. 1998;177(3):642-50.

28. Germon $\mathrm{P}$, Chen $\mathrm{YH}$, He L, Blanco JE, Bree A, Schouler C, Huang SH, MoulinSchouleur M. ibeA, a virulence factor of avian pathogenic Escherichia coli. Microbiol-Sgm. 2005;151:1179-86.

29. Rijavec M, Muller-Premru M, Zakotnik B, Zgur-Bertok D. Virulence factors and biofilm production among Escherichia coli strains causing bacteraemia of urinary tract origin. J Med Microbiol. 2008;57(11):1329-34.

30. Clermont O, Bonacorsi S, Bingen E. Rapid and simple determination of the Escherichia coli phylogenetic group. Appl Environ Microbiol. 2000; 66(10):4555-8.

31. Wang Y, Zhu J, Lu C, Wu B, Liu D, Hang W, Liu H, Liu X. Evidence of circulation of an epidemic strain of Pasteurella multocida in Jiangsu, China by multi-locus sequence typing (MLST). Infect Genet Evol. 2013;20:34-8.

\section{Submit your next manuscript to BioMed Central and we will help you at every step:}

- We accept pre-submission inquiries

- Our selector tool helps you to find the most relevant journal

- We provide round the clock customer support

- Convenient online submission

- Thorough peer review

- Inclusion in PubMed and all major indexing services

- Maximum visibility for your research

Submit your manuscript at www.biomedcentral.com/submit
) Biomed Central 